\title{
Research on Korean Hanja and Teaching Chinese vocabulary in CFL
}

\author{
Yan LIU, Mengxi JIA \\ College of Humanities \& Law, North China Univ. of Tech., Beijing, China (jljaly @ 163.com)
}

\begin{abstract}
The Korean Hanja comes from Chinese and many of them preserve their original Chinese word meaning. Because of the historical development and different culture background, a lot of differences are produced between the Korean Hanja and their corresponding Chinese. Based on an investigation of the new HSK vocabulary (third level), this paper attempts to find out the impact of Korean Hanja on the acquisition of Chinese vocabulary by Korean learners of Chinese.
\end{abstract}

Keywords-Korean Hanja, comparative analysis, vocabulary teaching, teaching suggestions

\section{韩语汉字词与对韩汉语词汇教学}

刘妍 贾梦溪

北方工业大学文法学院, 北京, 中国

摘 要 韩语汉字词被广泛运用在各个领域, 成为韩语的重要组成部分。学习汉语的韩国学生数量在不断增加, 与欧美国家的学 习者相比, 他们有不同的学习特点, 因此应该采用不同的教学策略。韩语中的汉字词对韩国学生学习汉语有积极和消极的影响。本文 分析了新 HSK 大纲中的三级词汇, 将其与对应的韩语汉字词进行比较, 在此基础上提出了教学建议。

关键词 韩语汉字词, 对比分析, 词汇教学, 教学建议

\section{1. 研究背景和目的}

据教育部网站公布的统计结果, 来自韩国的留学生数 量位于来华留学生数量按国别排序的第一位, 且这一数量 目前还在增长中。可见, 在国内韩语背景学习者成为了学 习汉语的最大团体。但是, 在目前的对外汉语教学实践中, 这些学生的韩语背景似乎并未得到足够的重视。

韩语属于黏着语, 从语法结构来看与汉语的孤立语语法结 构完全不同。但是, 在词汇方面, 韩语词汇与现代汉语词 汇却颇有洲源。从韩语的词汇构成来看, 韩语中有固有词、 汉字词和外来词。其中汉字词所占比例最高。韩国 HNGEUL 学会编纂的《大辞典》(1961) 所收 164125 条词语中, 汉 字词占 $52.11 \%$, 李熙升编纂的《国语大辞典》(1961) 所 收 275854 条词语中, 汉字词占 $69.32 \%$ 。所谓 “汉字词” 就是在韩国人的语言生活中, 用韩国人的发音习惯, 表达 在韩国语语法结构中的, 从中国来的词汇。书写这样的词 汇所用的韩文背后往往对应一个到多个汉字。韩语背景的 汉语学习者在学习汉语词汇过程中, 很容易受到其母语中 汉字词的影响, 将韩语汉字词的意义用法迁移到汉语中来。
这可以说是他们学习汉语词汇的一大优势, 但同时也是偏 误产生的原因。

\section{2. 研究内容和方法}

本文分析了新 HSK 大纲的三级词汇, 之所以选择三级 词汇, 是因为其中既有比较简单的名词、动词, 又有比较 抽象的各类词汇, 能够全面反映汉字词的特点。对于异同 的比较, 因为汉语与韩语属于不同语系, 语法差异较大, 所以本文只对词语的意义进行比较, 没有必要时不涉及语 法功能。对于韩语汉字词与其所对应的汉字的讨论, 本文 忽略繁简体汉字的字形差异, 只要汉语汉字词所对应的繁 体字能与现代汉语的简体字对应, 则视为字形相同。

本文中所涉及的汉语词汇释义参考《现代汉语词典》 (第五版), 韩语词汇释义参考《韩汉大辞典》。将词语的 义项进行比较分析, 根据词形词义分类, 再根据不同类别 研究其对于对韩汉语词汇教学的影响。 


\section{HSK 三级词汇分类整理分析}

\section{1 词形词义都基本相同}

词形词义都基本相同的词共有 63 个, 在 HSK 三级词汇 中所占比例为 $36.84 \%$ 。

“词形词义都基本相同” 的 “词形” 指汉字的形态。 汉语没有形态变化, 汉字的形态即汉字本身的样子。对于 韩语汉字词来说, 词形即韩语汉字词的韩文所对应的的汉 字。如 “북” 对应的汉字是 “北”, “방” 对应的汉字是 “方”, “북방” 即对应 “北方”。又如 “도人l”对应 “都市”; “청소” 对应 “清掃”。如果一个韩语汉字词的词义与其所对应的汉 字在现代汉语中的词义基本相同, 那么它就是 “词形词义 都基本相同”的词汇。

如果详细分析这些词汇, 他们大概又可以分为绝对同 义词和相对同义词。相对同义词指的是虽然词的理性意义 相同, 但词汇的情感、语体色彩等在两种语言中有不尽相 同的地方。相对同义词虽然会在一定程度上引发偏误, 但 主要发生在售贬误用、口语书面语误用方面。这样的差异 是因两国不同的自然地理条件及政治、文化因素影响产生 的。绝对同义词则是词语的词形、词义、感情色语体色彩 等都没有区别的词。这类词主要为简单的名词, 也有一部 分是简单的形容词和动词。绝对同义词本身是不容易引发 偏误的, 除非涉及到韩语汉字词的同音词现象, 即一个韩 语词汇对应多个汉语词汇。例如: “필수” 对应 “必須”与 “必需”。这样的情况会在汉字习得过程中造成一定的障 碍。

\section{2 词形相同词义部分相同}

所谓 “词形相同词义部分相同” 的词汇是指韩语汉字 词的词义与其所对应的汉字在现代汉语中的词义有相同的 部分也有不同的部分。经过社会生活的变化, 韩语汉字词 与现代汉语词汇之间就产生了差异。但是，因为二者同源， 其根本的、原始的意思还是能够反映在现代通用的意义中, 因此既有差异也有相同之处。经过词义演变，现在细分词 形相同词义部分同的词汇大致可以分为四种情况：汉语意 义范围大于韩语意义范围; 韩语意义范围大于汉语意义范 围汉语与韩语意义范围部分相同部分相异; 同形完全异义。 第四种情况比较少见。

\section{2.1 汉语意义范围大于韩语意义范围}

所谓 “汉语意义范围大于韩语意义范围” 就是韩语汉 字词所对应的的汉语词汇在现代汉语中的意义范围比这个 韩语词汇大。如 “经验”一词在汉语中的义项有 (1) 名 词, 由实践得来的知识或技能; (2) 动词, 经历; 体验。
而 “경험 (經驗) 하다” 只有作为动词 “经历; 体验” 的 意义。

3. 2.2 韩语意义范围大于汉语意义范围

“韩语意义范围大于汉语意义范围” 是指韩语汉字词所 对应的的汉语词汇在现代汉语中的意义范围比这个韩语词 汇要小。例如: “锻炼”一词在汉语中的意义有三项（1） 指锻造或冶炼;（2）通过体育运动使身体强壮; (3) 通过 生产劳动社会斗争和工作实践，使觉悟和工作能力提高。 而 “단련 (鍛煉) 하다” 的意义除了包括冶炼; 体育锻炼; 磨练、砥砺外还有一个 “锻炼” 所不包含的意义, 即 “练 习”。

\section{2 .3 汉语与韩语意义范围部分相同}

“汉语与韩语词汇部分相同” 是指韩语汉字词与其对应 的汉语词汇在词义范围上既有相同的部分又有不同的部 分。这种现象是由于同一个词汇在两个国家的语言中保留 了不同的古意或产生了不同的衍生义而产生的。例如: “敢” 在汉语中有如下义项: (1) 有勇气, 有胆量; (2) 助动词, 表示有胆量做某种事情;（3）助动词, 表示有把握做某种 判断; (4) 〈书面语〉谦辞, 表示冒昧的请求别人。而与之 对应的韩语汉字词 “감（敢）히” 在韩语中有两个义项,

（1）谦辞, 冒昧, 斗胆, 贸然; (2) 竟然敢。“감（敢） 히” 没有 “敢” 所有的 “有胆量做某事; 有把握做某种判 断” 的义项。而 “敢” 没有 “竟然敢” 的含义。虽然在一 些特殊的情境下汉语口语里有时也用 “敢” 表达 “竟然敢” 但是要用一定的语气来辅助, 还要在特定的语境中, 落实 到书面语还是要用 “竟然敢” 来表达。

\section{3 同形完全异义}

韩汉同形完全异义的词比较少见, HSK 三级词汇中有 “新闻”与 “众定 (新聞)”。汉语中 “新闻” 指报社、通 讯社、广播电台、电视台等报道的消息或社会上最新发生 的事情。而 “甾呈 (新聞)” 在汉语中一般指报纸。还有 “必 须”与 “필수 (必須)”。“필수 (必須)” 更倾向于汉语词 “必需” 的意思。“必须” 在翻译时常与固有词 “반드시” 互译。

\section{4 词形不同词义基本相同}

这一部分韩语汉字词所对应的汉字与该词所翻译成的 汉语词语用不同的语素或不同的语素构成方式来表达相同 的意思。造成这一现象的原因是多方面的。从韩语汉字词 的来源方面来看, 有从不同时期引自汉语词汇的词语; 有 韩国根据汉字自己造的汉字词, 如 “내외 (內外)” 指夫妻, 古代夫妇以 “内人”、“外子” 作为代词, 指代对方, 韩语 
据此造出了 “내외 (内外)”。韩语汉字词中还有一部分是借 用日语汉字词的词语。日语汉字词与汉语词汇的差异造成 了这些词汇的韩汉差异。如 “人진 (寫真)” 韩语与日语都 有 “照片” 的意思, 而汉语中只有在表示画或拍摄的人像 时才用 “写真”，一般只用 “照片”。

\section{4.1 部分构词语素同}

在词形不同的构词语素中, 有一部分词汇的构词语素 并不是完全不同, 例如: “别人”与 “타인 (他人)” 都是 人称代词, 指与 “自己” 相对的, 另外的人。二者都是偏 正短语, 分别用 “他” 和 “别” 这两个词根修饰限制 “人” 这个词根。而 “他人” 本身也是汉语词语, 与 “别人” 同 义, 只是 “他人” 书面语色彩更浓些, 而韩语中没有与 “别 人” 对应的汉字词, 口语中表达 “别人” 的意思也用 “타인 (他人)”。

\section{4.2 全部构词语素不同}

构词语素完全不同的词汇也可能有相同的意义。这类 韩语词所对应的汉字常常本身也是汉语词汇, 但韩汉之间 的意义有差别, 像这样的词和与其同形的汉语词也可以看 做同形异义词。例如 “河” 与 “江” 在汉语里从使用习惯 方面看是不同的。“亚马逊河” 就不能叫做 “亚马逊江” 而 “강（江）” 虽然对应的汉字是 “江” 但他它与 “江” “河” 都同义。在具体的翻译中, 按照习惯, 常把 “河” 译成 “강” 如 “아마존강（江）” 要翻译成 “亚马逊河”。

\section{4.3 构词语素词素相同位置不同}

韩汉同素逆序词是一个很复杂的现象。逆序汉字词与 其所对应的汉语词汇之间意义有可能相同, 有可能不同。 在词义基本相同的情况下又分不同情况。

在 HSK 三级词汇中，同素逆序的汉字词只有一个, “搬 运” 与 “운반 (運搬) 하다 ” 二者意义基本相同, 都是指 “把物品从一个地方运到另一个地方。”

\section{4. 韩语汉字词对韩国汉语学习者的影响}

\section{1 汉字词的积极影响}

韩语词汇在表达事物、名称、方位等生活实用名词中 汉字词所占比重很大。对于这一部分常用名词, 学生的学

习难度是比较小的。 三级词汇基本属于较简单的词汇, 由上文的统计分析可知, 在三级词汇中, 同形同义词占了 相当的比重, 这些词汇只需要简单的讲解就可以让学习者 理解记忆, 利用好这些词汇可以帮助学生快速扩大词汇量。 且这部分词汇由于与学习者的母语接近, 很容易拉近与学 习者的心理距离。消除使用表音文字的学习者初见象形文 字时产生的畏难情绪。

\section{2 汉字词的消极影响}

韩语汉字词与现代汉语的词汇差异给两种语言背景的 学习者学习目的语带来了很大的困扰。

同形同义词即上文所说的词形和词义都基本相同的 词。对于词形词义绝对相同的词, 基本上产生正迁移。而 相对同义词就非常容易产生负迁移。由于这些词词形相同, 词的理性意义也相同, 所以学习者很难意识到他们在用法、 褒贬、语体等方面的差异，容易误用且不容易被纠正。

同形异义词包括上文所说的词形相同词义部分相同的 词和同形完全异义词。由于词形相同, 很大一部分词义也 相同, 学习者很容易记住这些词, 但因为用法和意义的一 些差异, 学习者又很容易产生负迁移。这是他们口语、造 句和写作上词汇误用的一个主要原因。

\section{5. 与韩语汉字词相关的教学建议}

对外汉语教学专家陆剑明先生曾说: “一个外国学生要 学好汉语, 重要的是掌握大量的词汇, 因此词汇教学应该 是一个重点。” 对于韩语背景的汉语学习者来说, 韩语汉字 词对于对韩汉语教学的影响应该被给予足够的重视。充分 利用韩语汉字词能够帮助学生尽快扩大词汇量, 理解掌握 好汉语词汇。

\section{1 以汉字词为桥梁, 引导学习者了解汉字}

汉字是汉语词汇的基础, 也是汉字词的源本。它是韩 二者之间相通的核心。可以先通过一些简单的同形基本同 义词的韩汉对应来引导学习者认识汉字。在学习者认识到 了这种字与字的对应之后, 可以进一步向他们解释汉字的 字义。

在认识到汉字音形义三者的结合之后, 就可以把字代 入词语中, 具体学习词汇了。虽然现代汉语中的汉字与韩 语汉字词中的汉字在意义用法上有着各种各样的差别, 但 是利用汉字词可以缓解学习者的畏难情绪, 帮助他们尽快 熟悉汉字及汉字词语。而同形同义词对应记忆又可以有效 的帮助他们扩大词汇量。

\section{2 辨析差异, 减少偏误}

同形异义词在初次使用时偏误率较高。由于对韩汉语 义范围差异的不当把握, 对于汉语意义范围小于韩语汉字 词意义范围的词汇和二者意义范围有交叉的词汇会发生词 语误代的情况。在教学中, 要让学习者意识到韩汉是有所 差别的。由于这种偏误的发生随机性很高, 所以在教学过 程中要尽量让学习者多造句, 以便把那些可能隐藏的偏误 及时发现、纠正。对于汉语词汇范围大于韩语词汇范围的 
词, 学习者对于它的那些在韩语里没有的用法会感到困惑, 不确定对不对, 也不敢使用, 这就需要在学习过程中不断 积累。教师可以总结和辨析常见的短语搭配, 便于学习者 学习掌握。

本文为 “北京高校青年英才计划 (Beijing Higher Education Young Elite Teacher Project YETP1432) 阶段 性成果

\section{参考文献(References)}

[1] Ellis. R, Second Language Acquisition. Oxford: Oxford
University Press, 1997.

[2] Quan Xianglan, The impact of Korean Hanja on the acquisition of Chinese vocabulary by Korean learners, Chinese Teaching in the World, 2006 (1).

[3] S. P. Corder, the Significance of Learner's Errors. Penguin: International Review of Applied Linguistics, 1967, (4).

[4] S. P. Corder, Introducing Applied Linguistics. Harmondsworth: Penguin Books Ltd, 1973.

[5] Zhang Guangjun, Contractions of the Words Written in Chinese Characters in Korean, Chinese Language Learning, 2006 (4). 